\title{
Fast Pattern Classification of Ventricular Arrhythmias Using Graphics Processing Units
}

\author{
Noel Lopes ${ }^{1,2}$ and Bernardete Ribeiro ${ }^{1}$ \\ 1 CISUC - Center for Informatics and Systems of University of Coimbra, Portugal \\ ${ }^{2}$ UDI/IPG - Research Unit, Polytechnic Institute of Guarda, Portugal \\ noel@ipg.pt, bribeiro@dei.uc.pt
}

\begin{abstract}
Graphics Processing Units (GPUs) can provide remarkable performance gains when compared to CPUs for computationally-intensive applications. In the biomedical area, most of the previous studies are focused on using Neural Networks (NNs) for pattern recognition of biomedical signals. However, the long training times prevent them to be used in real-time. This is critical for the fast detection of Ventricular Arrhythmias (VAs) which may cause cardiac arrest and sudden death. In this paper, we present a parallel implementation of the Back-Propagation (BP) and the Multiple Back-Propagation (MBP) algorithm which allowed significant training speedups. In our proposal, we explicitly specify data parallel computations by defining special functions (kernels); therefore, we can use a fast evaluation strategy for reducing the computational cost without wasting memory resources. The performance of the pattern classification implementation is compared against other reported algorithms.
\end{abstract}

Keywords: GPU Computing, Parallel Programming, Neural Networks.

\section{Introduction}

Neural networks (NNs) have been successfully applied as pattern recognition systems in many areas [1]. However building a NN solution, is usually a computationally expensive task, demanding a considerable amount of time. Depending on the complexity of the problem, in most cases several NNs, with different configurations, must be trained before achieving a good solution. Thus the time required to train the NNs alone may prevent high quality solutions from being found. Dedicated hardware can be used to overcome this problem. Nevertheless this solution is often not chosen due to its high cost and reduced flexibility [1.

Graphics Processing Units (GPUs) can offer a more flexible and economical alternative to the use of dedicated hardware, yet a powerful one. Originally GPUs were developed as specialized accelerators for triangle rasterization. The transition to general purpose engines, aiming at high throughput floating-point computation, is witnessed by GPU implementations of machine learning algorithms 34. At present computers possess a GPU that offers increasing degrees of programmability allowing enough flexibility to be used to accelerate nongraphics applications [5]. GPUs are much more effective in utilizing parallelism

E. Bayro-Corrochano and J.-O. Eklundh (Eds.): CIARP 2009, LNCS 5856, pp. 603 610, 2009.

(C) Springer-Verlag Berlin Heidelberg 2009 
(and pipelining) than general purpose CPUs [6]. Due to its inherent parallel architecture, GPUs can provide remarkable performance gains when compared to CPUs for computationally-intensive applications. Thus they provide an attractive alternative to use dedicated hardware in machine learning, namely in the NN field [5]. Moreover, GPUs still service the large gaming industry and so they are relatively inexpensive [3]. However, until recently, General-Purpose computation on the GPU (GPGPU), required the programmer to master the fundamentals of graphics shading languages that require prior knowledge on computer graphics 6. This changed when NVIDIA introduced a new data-parallel, C-language programming API called CUDA (Compute Unified Device Architecture) that bypasses the rendering interface and avoids the difficulties of classic GPGPU 46 .

The rest of this paper is organized as follows. Section 2 introduces the CUDA programming model and its architecture. Section 3 summarizes the Multiple Back-Propagation (MBP) algorithm, whose GPU parallel implementation is discussed latter on section 4 . Section 5 details the steps taken to create NNs capable of detecting VAs based on time and frequency domain features obtained from electrocardiography's (ECGs). Section 6 compares the GPU and CPU implementations of the algorithms. Finally, section 7 summarizes contributions and addresses directions for future work.

\section{CUDA Programming Model and Architecture}

The CUDA programming model extends the $\mathrm{C}$ language, allowing the programmer to explicitly specify data parallel computations by defining special functions, named kernels. Kernels are executed in parallel by different CUDA threads, on a physically separate device (GPU) that operates as a co-processor to the host (CPU) running the program. Figure 1 shows an example of a simple Kernel. Threads are organized into blocks that are required to execute independently. To invoke a kernel, programmers use language extensions in order to specify the runtime values for the number of blocks (organized into a two dimensional grid) and the number of threads per block [7]. The CUDA programming model is supported by an architecture built around a scalable array of multi-threaded Streaming Multiprocessors (SMs). Each SM has eight Scalar Processor (SP) cores. When a program on the host invokes a kernel grid, its blocks are enumerated and distributed to SM with available execution capacity. As thread blocks finish their execution, new blocks are launched on the vacated SMs. Each

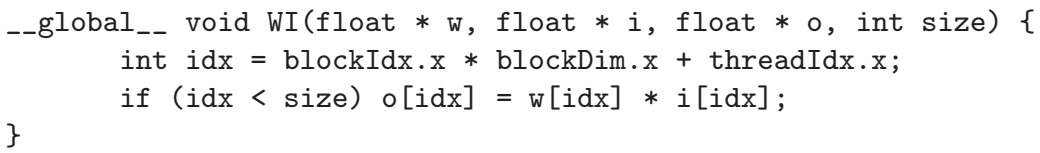

Fig. 1. Kernel that multiplies each element of the vector $\mathbf{w}$ by the corresponding element of the vector $\mathbf{i}$ placing the result on the vector $\mathbf{o}$ 
SM creates, manages, and executes concurrent threads in hardware with zero scheduling overhead and can implement fast barrier synchronization. These are the keys to efficiently support fine-grained parallelism [7.

\section{Multiple Back-Propagation}

Multiple Back-Propagation (MBP) is a generalization of the Back-Propagation (BP) algorithm that can be used to train Multiple Feed-Forward (MFF) networks [8]. Jointly MFF networks and the MBP algorithm shape an architecture that is (in most situations) preferable to the use of feed-forward (FF) networks trained with the BP algorithm. MFF networks are obtained by integrating two FF networks (a main network and a space network) as shown in Figure 2 [9]. The main network contains at least one selective activation neuron. Selective activation neurons differentiate between stimulus (patterns). Their response depends on the space localization of a pattern $p$ presented to the network and might be amplified or reduced accordingly. Its output is given by (1):

$$
y_{k}^{p}=m_{k}^{p} \mathcal{F}_{k}\left(a_{k}^{p}\right)=m_{k}^{p} \mathcal{F}_{k}\left(\sum_{j=1}^{N} w_{j k} y_{j}^{p}+\theta_{k}\right),
$$

where $y_{k}^{p}$ is the output of neuron $k, m_{k}^{p}$ the importance of the neuron, that varies accordingly to the pattern (stimulus) presented to the network, $\mathcal{F}_{k}$ the neuron activation function, $a_{k}^{p}$ its activation, $\theta_{k}$ the bias and $w_{j k}$ the weight of the connection between neuron $j$ and neuron $k$. The importance $\left(m_{k}^{p}\right)$ of each neuron $k$ for the current pattern $p$ is determined by a standard FF network, that receives the same inputs as the main network, named space network because it is implicitly dividing the input space. The main network can only calculate its outputs after knowing the outputs $\left(m_{k}^{p}\right)$ of the space network. Thus the two networks will function in a collaborative manner and must be trained together.

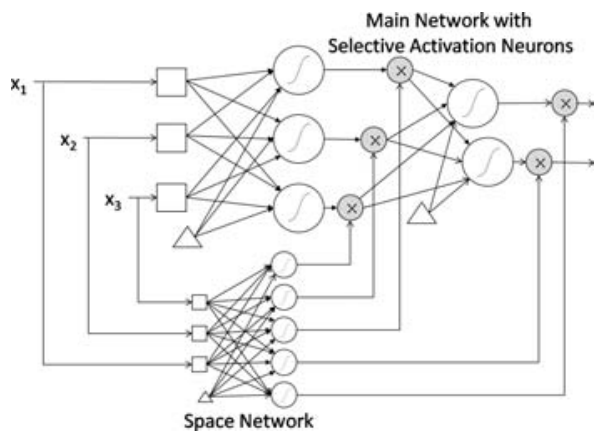

Fig. 2. MFF Network. Squares represent input neurons, white circles hidden and output neurons, gray circles multipliers and triangles the bias. 


\section{BP and MBP Parallel GPU Implementation}

In this phase we choose to implement exclusively the batch training mode for both the BP and MBP algorithms, since this is the mode that can benefit the most from a parallel implementation. The resulting implementation exploits the widely used adaptive step size technique due to stability of the algorithms and improved training speeds. In order to simultaneously implement both algorithms, five kernels listed on Table1 1 were created. Figure 3illustrates the steps necessary to train, during one epoch, a MFF network comprising a main network containing an input layer with $N_{i}$ neurons, a hidden layer with $N_{h}$ neurons with selective activation and an output layer with $N_{o}$ neurons (without selective activation) and a space network containing an input layer with $N_{i}$ neurons and an output layer with $N_{h}$ neurons. This is the configuration we will use later on section 6 to train MFF networks for the VA problem. It is assumed the training set contains $N_{p}$ patterns. First FireLayer is called with the network inputs vector $\mathbf{x}$ and the weights vector of the space network $\mathbf{w}_{\mathbf{s}}$. As a result a vector $\mathbf{m}$ containing the importance of each neuron with selective activation, for each pattern, is calculated. This vector is then used together with $\mathbf{x}$ and the vector containing the input weights of the main network hidden layer $\mathbf{w}_{\mathbf{h}}$ to call FireLayer in order to calculate the hidden layer outputs $\mathbf{y}_{\mathbf{h}}$. To complete the calculation of the network outputs $\mathbf{y}$, FireOutputLayer is then called using $\mathbf{y}_{\mathbf{h}}$, the input weights of the output layer $\mathbf{w}_{\mathbf{o}}$ and the desired outputs vector $\mathbf{d}$. This will also calculate the local gradients of the output layer $\delta_{\mathbf{o}}$. At this time the Root Mean Square (RMS) error of the network can be calculated by calling the CalculateRMS kernel. Then CalcLocalGradients is used to determine the local gradients of the hidden layer $\delta_{\mathbf{h}}$ and the local gradients of the space layer $\delta_{\mathbf{s}}$. Finally CorrectWeights is called several times to adjust the weights $\mathbf{w}_{\mathbf{s}}, \mathbf{w}_{\mathbf{h}}$ and $\mathbf{w}_{\mathbf{o}}$ of the MFF network. Figure 4 shows the percentage of time spent by the GPU in each Kernel for the VA problem.

Table 1. Kernels used to implement both the BP and the MBP algorithms. The Kernels shown here process all the training patterns simultaneously.

\begin{tabular}{ll}
\hline Kernel & Purpose \\
\hline FireLayer & $\begin{array}{l}\text { Calculates the outputs of all neurons in a given layer. } \\
\text { FireOutputLayer } \\
\text { Calculates the outputs of the NN output layer. If the layer } \\
\text { contains selective activation neurons, the local gradients of } \\
\text { the corresponding space network neurons are also calculated. }\end{array}$ \\
CalcLocalGradients & $\begin{array}{l}\text { Calculates the local gradient of all neurons in a hidden layer. } \\
\text { If there are selective activation neurons, the local gradients of } \\
\text { the corresponding space network neurons are also calculated. }\end{array}$ \\
CorrectWeights & $\begin{array}{l}\text { Adjust the weights of a given layer. } \\
\text { CalculateRMS }\end{array}$ \\
Calculate the Root Mean Square (RMS) error of the network.
\end{tabular}




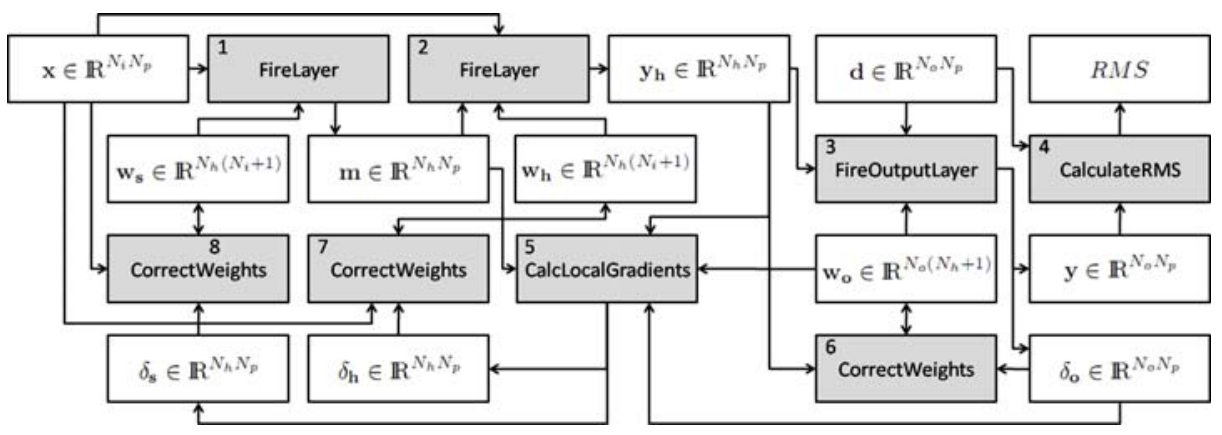

Fig. 3. Model of the sequence of kernels launched by the host (in each epoch) to train a MFF network comprising a main network with 3 layers and a space network with 2 layers (that calculates the importance of the hidden neurons of the main network). White rectangles represent input and output vectors, whilst gray rectangles represent kernels.

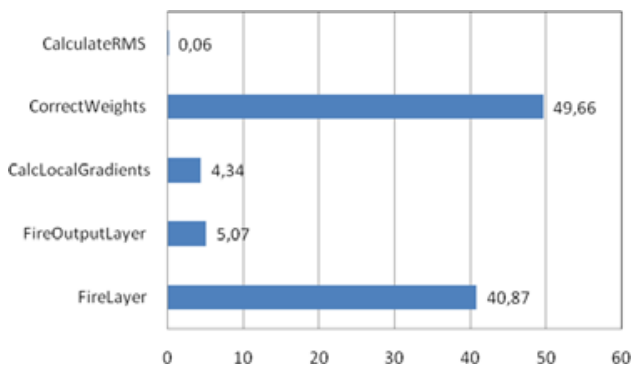

Fig. 4. Percentage of time spent by the GPU in each Kernel

\section{$5 \quad$ Ventricular Arrhythmias Assessment}

Nowadays most countries face high and increasing rates of cardiovascular diseases. In Portugal there is a $42 \%$ probability of dying of these diseases and worldwide they are accountable by 16.7 million deaths per year [10. In this context VAs assume a significant role given that their prevalence can lead to life threatening conditions that may result in cardiac arrest and sudden death. VAs evolve from simple Premature Ventricular Contractions (PVCs) which are usually benign, to ventricular tachycardia and finally to critical ventricular fibrillation episodes which are potentially fatal and the main cause of sudden cardiac death. The detection of PVCs from an ECG is thus of major importance, since they are associated with an increased risk of adverse cardiac events. A typical ECG tracing of a ordinary heartbeat consists of a $\mathrm{P}$ wave, a QRS complex and a T wave (see figure 5 a). PVCs result from an ectopic depolarization on the ventricles, which results on a wider and abnormally shaped QRS complex. Moreover, typically QRS complexes are not preceded by $\mathrm{P}$ waves, and $\mathrm{T}$ waves are usually larger and with opposite deflection to the QRS complex. For high-performance detection of VAs we take advantage of the power of GPUs to significantly 


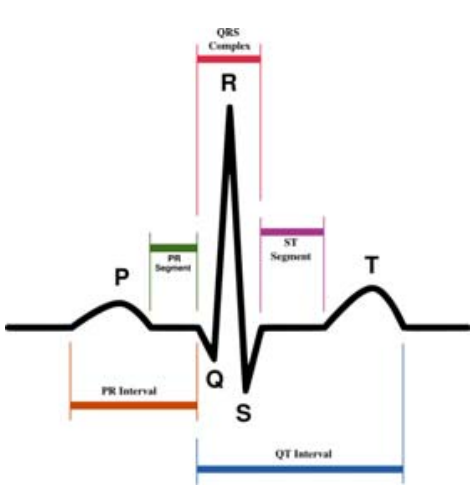

\begin{tabular}{ll}
\hline Feature & Description \\
\hline RRav & RR mean interval \\
RR0 & Last RR interval \\
SN & Signal/Noise estimation \\
Q1 & Q-wave length \\
(Qcx, Qcy) & Q-wave mass center (x,y) coordinates \\
(Qpx, Qpy) & Q-wave peak (x,y) coordinates \\
Rl & R-wave length \\
(Rcx, Rcy) & R-wave mass center (x,y) coordinates \\
(Rpx, Rpy) & R-wave peak (x,y) coordinates \\
Sl & S-wave length \\
(Scx, Scy) & S-wave mass center (x,y) coordinates \\
(Spx, Spy) & S-wave peak (x,y) coordinates \\
\hline
\end{tabular}

Fig. 5. (a) Schematic diagram of normal sinus rhythm for a human heart as seen on ECGs. (b) Selected features from the ECG signal.

accelerate the training of a NN based approach. The NNs take as inputs 18 features (see figure 5b) that were chosen in [1112. For comparison, we also use the same training, test and validation datasets, each one containing 19391 samples 1

\section{Results for the CPU and GPU Implementations}

In order to compare the performance of the GPU and the CPU versions we respectively used $(i)$ the proposed CUDA implementation (see section 4) and (ii) the Multiple Back-Propagation software. Multiple Back-Propagation is a highly optimized software, developed in $\mathrm{C}++$, for training $\mathrm{NNs}$ with the BP and MBP algorithms 2 The GPU version was benchmarked on two different NVIDIA devices: a GeForce 8600 GT with 4 SM (32 cores) and a GTX 280 with $30 \mathrm{SM}$ (240 cores). The CPU version was benchmarked on a Intel Core 2 $6600 \mathrm{CPU}(2.4 \mathrm{GHz})$. Results were obtained, using the VA datasets, both for the $\mathrm{BP}$ and the MBP algorithms. The FF networks trained consisted of 3 layers. As for MFF networks the topology was described in section 4 (see Figure 3 description). Experiments demonstrate that the GPU implementation delivers considerable speedups comparatively to the CPU. Figure 6 shows the number of epochs trained per minute accordingly to the hardware. Using the GTX 280 GPU it is possible to reduce the training time more than 50 times relatively to the CPU, as shown in Figure 7. It is interesting to note that as the number of hidden neurons increases so does the gain of speed provided by the GPU, because more processing that can be parallelized is required. Since currently our implementation does not support cross validation, preliminary tests were conducted in order to determine when to stop training. Based on the information collected we decided to train both FF and MFF networks during 1 million

\footnotetext{
${ }^{1}$ MIT-BIH Arrhythmia Database (http://www.physionet.org/physiobank/)

${ }^{2}$ Multiple Back-Propagation software is freely available at http://dit.ipg.pt/MBP
} 

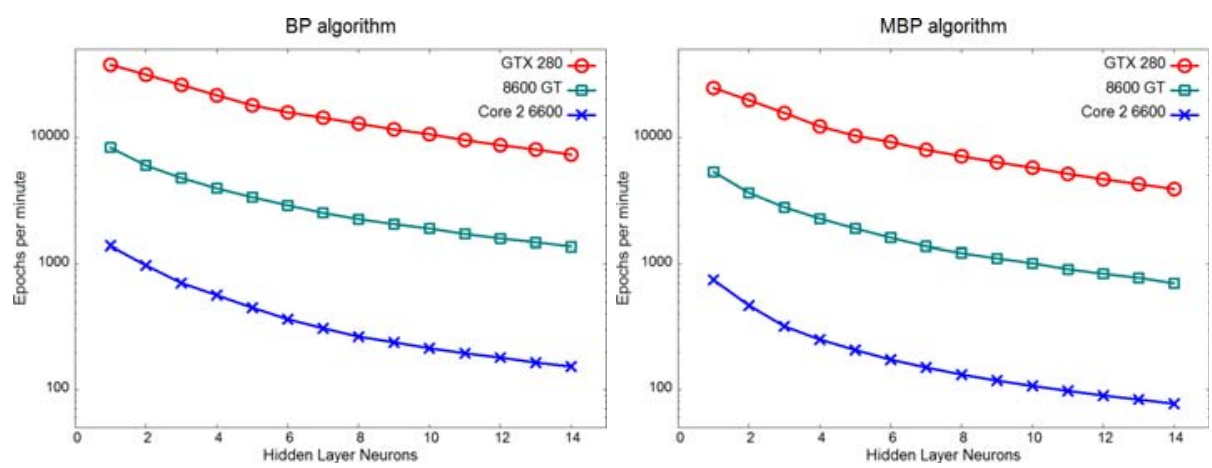

Fig. 6. Number of epochs trained per minute, accordingly to the hardware

Table 2. PVC detection: performance results of the NNs

\begin{tabular}{lcccccc}
\hline Metrics & \multicolumn{3}{c}{ BP (FF) } & \multicolumn{3}{c}{ MBP (MFF) } \\
& Train & Test & Val & Train & Test & Val \\
\hline Sensitivity & 98.07 & 95.94 & 94.67 & 97.42 & 95.54 & 94.47 \\
Specificity & 99.84 & 99.62 & 99.61 & 99.87 & 99.68 & 99.70 \\
Accuracy & 99.70 & 99.33 & 99.23 & 99.68 & 99.36 & 99.30 \\
\hline
\end{tabular}

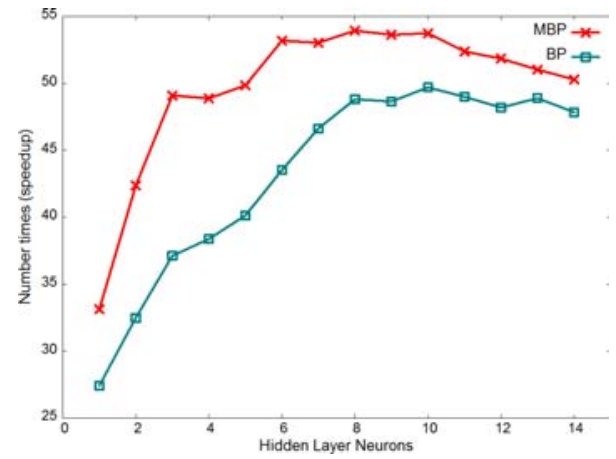

Fig. 7. Increase in speed provided by the GTX 280 relatively to the CPU

epochs, varying the number of hidden neurons. It is worth to mention that during the preliminary tests some NN were trained up to 3 million epochs, requiring almost 9 hours of train on a GTX 280 GPU. We estimate that if such NNs were trained on the CPU we would need almost 3 weeks to train each one. Table 2 shows the performance results of the best networks found, trained with the BP and the MBP. The best network trained with the BP algorithm has 14 hidden neurons and the best trained with the MBP has 13 hidden neurons with selective activation. The results, which improve over those previously obtained in [112, could not be obtained without the gain of speed provided by the GPU. 


\section{Conclusion}

In this paper, the parallel implementation of MBP (and BP) algorithms to train MFF neural networks has proved highly efficient for pattern classification. Results confirm that the GPU can provide a more flexible and cheap alternative to the use dedicated hardware in the NN field. The speedups attained, which are already impressive, are expected to increase even more, as new GPUs containing a greater number of cores arrive to the market. This allows for researchers and practitioners in pattern recognition to implement high quality NN solutions that could be disregarded otherwise, due to temporal and financial constraints. In future work online implementation of both algorithms will be considered.

\section{References}

1. Brandstetter, A., Artusi, A.: Radial basis function networks GPU based implementation. IEEE Transactions on Neural Networks 19(12), 2150-2154 (2008)

2. Vonk, E., Jain, L.C., Veelenturf, L.P.J.: Neural network applications. In: Jain, L.C. (ed.) Electronic Technology Directions, pp. 63-67. IEEE Computer Society, Los Alamitos (1995)

3. Catanzaro, B., Sundaram, N., Keutzer, K.: Fast support vector machine training and classification on graphics processors. In: Proc. of the 25th International Conference on Machine Learning (ICML 2008), Helsinki, Finland, pp. 104-111 (2008)

4. Che, S., Boyer, M., Meng, J., Tarjan, D., Sheaffer, J.W., Skadron, K.: A performance study of general-purpose applications on graphics processors using CUDA. Journal of Parallel and Distributed Computing 68(10), 1370-1380 (2008)

5. Steinkrau, D., Simard, P.Y., Buck, I.: Using GPUs for machine learning algorithms. In: Proc. 8th Int. Conf. on Doc, pp. 1115-1119. IEEE Computer Society, Los Alamitos (2005)

6. Jang, H., Park, A., Jung, K.: Neural network implementation using CUDA and OpenMP. In: DICTA 2008: Proc. of the 2008 Digital Image Computing: Techniques and Applications, Washington, DC, USA, pp. 155-161. IEEE Comp. Society, Los Alamitos (2008)

7. NVIDIA CUDA Programming Guide Version 2.2 (2009)

8. Lopes, N., Ribeiro, B.: An efficient gradient-based learning algorithm applied to neural networks with selective actuation neurons. Neural, Parallel \& Scientific Computations 11(3), 253-272 (2003)

9. Lopes, N., Ribeiro, B.: Hybrid learning in a multi-neural network architecture. Neural Networks, 2001. In: Proc. of IJCNN 2001. Int Joint Conf. on Neural Networks, vol. 4, pp. 2788-2793 (2001)

10. WolframAlpha - computational knowledge engine, http://www . wolframalpha.com

11. Marques, A.: Feature extraction and PVC detection using neural networks and support vector machines. Master's thesis, University of Coimbra (2007)

12. Ribeiro, B., Marques, A., Henriques, J., Antunes, M.: Choosing real-time predictors for ventricular arrhythmia detection. IJPRAI 21(8), 1249-1263 (2007) 